\title{
OPEN Aberrantly higher functional connectivity in the salience network is associated with transient global amnesia
}

\author{
Geon Ha Kim ${ }^{1}$, Bori R. Kim ${ }^{1,2}$, Min Young Chun ${ }^{1}$, Kee Duk Park ${ }^{1}$, Soo Mee Lim ${ }^{3}$ \& \\ Jee Hyang Jeong ${ }^{4}$
}

Triple intrinsic brain networks including the salience network (SN), default mode network (DMN), and central executive network (CEN), are known to be important in human cognition. Therefore, investigating those intrinsic brain networks in transient global amnesia (TGA) may offer novel insight useful for the pathophysiology of TGA. Fifty TGA patients underwent the resting state functional magnetic resonance imaging (rsfMRI) within $24 \mathrm{~h}$, at $72 \mathrm{~h}$, and 3 months after TGA onset. Twentyfive age, gender matched controls also underwent rsfMRI. Within $24 \mathrm{~h}$ of TGA onset, TGA patients showed greater functional connectivity in the $\mathrm{SN}$ and lower functional connectivity in the DMN, while relatively preserved functional connectivity was observed in the CEN. Interestingly, TGA patients continued to show decreased connectivity in the DMN, while no alterations were shown in the SN $72 \mathrm{~h}$ after illness onset. Three months after TGA onset, alterations of functional connectivity in the SN or the DMN were normalized. Our findings suggest that TGA is associated with transient greater functional connectivity in the $\mathrm{SN}$ and lower connectivity in the DMN.

Transient global amnesia (TGA) is a clinical syndrome characterized by the sudden onset of anterograde and retrograde amnesia that lasts up to $24 \mathrm{~h}^{1}$. Although TGA patients exhibit anterograde amnesia for episodic memory, other memories, including semantic, procedural, and recognition memory, remain intact ${ }^{2}$. In addition, TGA patients do not exhibit focal neurological or other cognitive deficits such as attention. Several factors have been implicated in the pathophysiology of TGA, including migraines, focal ischemia, venous flow abnormalities, and epilepsy ${ }^{3}$. However, the etiology of TGA remains poorly understood.

Previous neuroimaging studies using diffusion-weighted imaging (DWI) have shown that approximately $70 \%$ of TGA patients exhibit focal hippocampal lesions in the cornus ammonis (CA) $1^{4,5}$. These hippocampal lesions developed between 24 and $48 \mathrm{~h}$ following the onset of TGA ${ }^{3,6}$. However, in a substantial number of patients with TGA, there were no visible DWI lesions of hippocamps even though they had typical symptoms of TGA ${ }^{3,6}$, which might suggest a threshold-dependent phenomenon of TGA that leads to functional deficits of the brain but does not lead to detectable signal changes on magnetic resonance imaging (MRI).

Recent advances in functional neuroimaging have permitted the investigation of large-scale functional brain networks. Previous research has suggested that the triple major brain network models are related to cognition ${ }^{7-10}$, which include the salience network (SN), default mode network (DMN), and central executive network $(\mathrm{CEN})^{11}$. It has been noted that the SN and CEN typically demonstrate increased connectivity in response to external salient events or tasks ${ }^{11,12}$, while the DMN is associated with episodic memory retrieval and autobiographical memory ${ }^{11,13,14}$. Previous research suggested that abnormal organization and functioning of those triple neurocognitive networks are prominent features of several neuropsychiatric disorders ${ }^{11}$.

Although there have been several functional neuroimaging studies of TGA, most studies have focused on the hippocampus and hippocampal networks ${ }^{15,16}$. Recent previous study with resting-state functional MRI (rsfMRI) in TGA patients has shown that decreased functional connectivity on memory network including hippocampus,

${ }^{1}$ Department of Neurology, College of Medicine, Ewha Womans University Mokdong Hospital, Ewha Womans University, Seoul, Republic of Korea. ${ }^{2}$ Ewha Medical Research Institute, Ewha Womans University, Seoul, Republic of Korea. ${ }^{3}$ Department of Radiology, Ewha Womans University Seoul Hospital, Ewha Womans University College of Medicine, Seoul, Republic of Korea. ${ }^{4}$ Department of Neurology, Ewha Womans University Seoul Hospital, Ewha Womans University College of Medicine, 1071, 260, Gonghang-daero, Gangseo-gu, Seoul 07804, Republic of Korea. ${ }^{\square}$ email: jjeong@ewha.ac.kr 


\begin{tabular}{|c|c|c|c|c|c|}
\hline Mean \pm SD & $\begin{array}{l}\text { Controls } \\
(\mathbf{n}=25)\end{array}$ & $\begin{array}{l}\text { TGA } \\
(n=50)\end{array}$ & $\begin{array}{l}\text { Hyperacute } \\
(n=26)\end{array}$ & $\begin{array}{l}\text { Post-acute } \\
(\mathrm{n}=24)\end{array}$ & P for group (Controls vs Total TGA) \\
\hline Age (years) & $60.2 \pm 5.8$ & $60.6 \pm 6.3$ & $60.8 \pm 6.8$ & $60.4 \pm 5.8$ & 0.76 \\
\hline Education (years) & $9.8 \pm 4.6$ & $11.2 \pm 3.6$ & $10.2 \pm 3.3$ & $12.3 \pm 3.7$ & 0.16 \\
\hline Female, n (\%) & $19(76.0)$ & $42(84.0)$ & $22(84.6)$ & $20(83.3)$ & 0.40 \\
\hline \multicolumn{6}{|l|}{ Risk factors } \\
\hline Hypertension, n (\%) & $4(16.0)$ & $14(28.0)$ & $5(19.2)$ & $9(37.5)$ & 0.25 \\
\hline Diabetes mellitus, n (\%) & $4(16)$ & $3(6.0)$ & $2(7.7)$ & $1(4.2)$ & 0.16 \\
\hline Hyperlipidemia, n (\%) & $3(12)$ & $10(20.0)$ & $4(15.4)$ & $6(25)$ & 0.39 \\
\hline Cardiac disease, $\mathrm{n}(\%)$ & $2(8.0)$ & $4(8.0)$ & $1(3.9)$ & $3(12.5)$ & 1.0 \\
\hline Stroke, n (\%) & $0(0)$ & $3(6.0 \%)$ & $0(0)$ & $3(12.5)$ & 0.21 \\
\hline Migraine, n (\%) & $0(0)$ & $7(14 \%)$ & $5(19.2)$ & $2(8.3)$ & 0.05 \\
\hline \multicolumn{6}{|l|}{ TGA symptoms } \\
\hline Duration of TGA symptoms (min) & NA & $497.8 \pm 313.5$ & $513.0 \pm 311.6$ & $481.3 \pm 321.7$ & NA \\
\hline $\begin{array}{l}\text { Time lapse from symptom onset to } \\
\text { MRI scan (min) }\end{array}$ & NA & $689.6 \pm 425.0$ & $420.8 \pm 231.1$ & $1137.5 \pm 260.3$ & NA \\
\hline K-MMSE (during ER visit) & $28.6 \pm 1.4$ & $23.1 \pm 3.2$ & $22.8 \pm 3.3$ & $23.4 \pm 3.2$ & $<0.001^{\star}$ \\
\hline Time orientation & $4.9 \pm 0.3$ & $2.3 \pm 1.6$ & $2.2 \pm 1.5$ & $2.4 \pm 1.8$ & $<0.001^{*}$ \\
\hline Place orientation & $5.0 \pm 0.2$ & $4.3 \pm 0.8$ & $4.2 \pm 0.8$ & $4.4 \pm 0.7$ & $<0.001^{*}$ \\
\hline Registration & $3.0 \pm 0$ & $3.0 \pm 0$ & $3.0 \pm 0$ & $3.0 \pm 0$ & NA \\
\hline Attention and calculation & $4.5 \pm 0.6$ & $4.0 \pm 1.1$ & $4.2 \pm 1.1$ & $3.8 \pm 1.2$ & NA \\
\hline Recall & $2.4 \pm 0.9$ & $0.9 \pm 1.0$ & $0.9 \pm 1.0$ & $1.1 \pm 0.9$ & $<0.001^{*}$ \\
\hline Language & $7.9 \pm 0.3$ & $7.7 \pm 0.5$ & $7.6 \pm 0.5$ & $7.7 \pm 0.5$ & 0.02 \\
\hline Interlocking pentagon drawing test & $1.0 \pm 0$ & $1.0 \pm 0.2$ & $1.0 \pm 0$ & $0.9 \pm 0.2$ & 0.43 \\
\hline K-MMSE (72 h) & NA & $28.8 \pm 1.2$ & $28.5 \pm 1.2$ & $29.0 \pm 1.2$ & NA \\
\hline K-MMSE (3 months) ${ }^{\mathrm{a}}$ & NA & $29.5 \pm 1.0$ & $29.2 \pm 1.3$ & $29.8 \pm 0.4$ & NA \\
\hline
\end{tabular}

Table 1. Demographic characteristics. ${ }^{a}$ Only 22 TGA patients were evaluated at 3 months after onset. K-MMSE Korean version of the Mini Mental State Examination, SD standard deviation, TGA transient global amnesia, NA not applicable, ER emergency room.

parahippocampal gyrus and posterior cingulate gyrus, which is more prominent in the hyperacute phase than the post-acute phase ${ }^{16}$. In addition, more recent study with resting state connectivity reported that TGA patients showed reduced functional connectivity in executive network such as frontal, parietal and insular cortex ${ }^{17}$. However, there were few studies that investigated alterations of triple intrinsic brain network in TGA patients. Given that up to $90 \%$ of TGA patients reported precipitating causes such as physical or emotional stress prior to the amnestic symptoms ${ }^{18}$, investigating intrinsic brain networks may offer novel insight that may be useful for examining the pathophysiology of TGA.

The aim of this study was to investigate whether TGA patients have alterations in the triple intrinsic brain networks in the SN, DMN, and CEN within $24 \mathrm{~h}$ as well as at $72 \mathrm{~h}$ and 3 months after symptom onset.

\section{Results}

Demographic characteristic. There were no significant demographic differences between the TGA and control groups regarding age, sex, and years of education (Table 1). There were no significant group differences in vascular risk factors such as hypertension, diabetes, cardiac disease, and history of stroke. TGA patients had more migraines compared to the controls; however, this was not statistically significant $(P=0.05)$.

The mean duration of TGA symptoms was $497.8 \pm 313.5 \mathrm{~min}$, and the mean time elapsed between the onset of TGA and the baseline MRI scan was $689.6 \pm 425.0 \mathrm{~min}$. Among 50 TGA patients, 26 patients were regarded as hyperacute phase, which referred to those who still had TGA symptoms such as temporal disorientation or repetitive questioning during the baseline MRI scan, whereas 24 TGA patients were regarded as the post-acute phase that referred to TGA patients who no longer exhibited repetitive questioning but still displayed memory deficits on MMSE. TGA patients had lower mean K-MMSE scores during the acute phase (22.7 \pm 3.3$)$ compared to follow-up visits, including at $72 \mathrm{~h}$ after illness onset $(28.6 \pm 1.4)$ and 3 months after illness onset $(29.5 \pm 1.0)$.

Emotional stress, including arguments with others and death of relatives or friends, precipitated 52\% of TGA cases. Physical activity, including heavy lifting, climbing, and swimming, precipitated 26\% of TGA cases (Fig. 1).

All 50 TGA patients with acute phase participated in the follow-up MRI $72 \mathrm{~h}$ after illness onset, while only 22 patients participated in the 3-month follow-up MRI scan. Among 50 patients with TGA who visited to ER, 34 (68.0\%) had a hyperintense lesion on the hippocampus according to DWI during the MRI examination at $72 \mathrm{~h}$ after illness onset. Clinical characteristics of participants who participated at 3-month follow up visit $(n=22)$ and of those who did not $(n=28)$ are shown in Table e-1. 


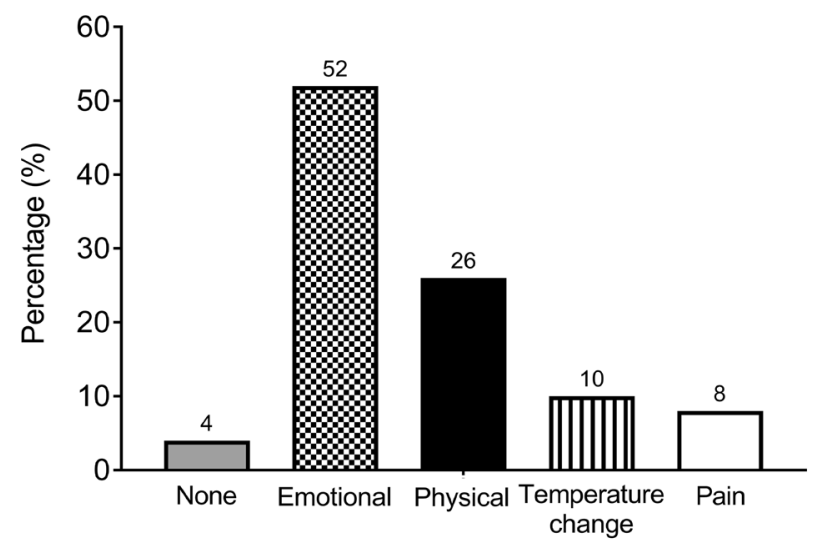

Figure 1. Percentage of precipitating factors in TGA patients. Emotional stress was reported by $52 \%$ of TGA patients. Emotional stress included arguments or physical fights with others and the death of a relative or friend. Physical activity, including heavy lifting, climbing, cycling, and swimming, precipitated 26\% of TGA cases.

Primary analyses. Alterations in functional networks during the acute phase of TGA. Within $24 \mathrm{~h}$ of illness onset, TGA patients showed greater functional connectivity in the SN but relatively lower functional connectivity in the DMN compared to controls (Fig. 2) after adjusting age and sex.

The topographical analysis demonstrated that TGA patients with acute phase showed greater functional connectivity, especially in the bilateral insular cortex and operculum, within the SN, but had lower functional connectivity in the bilateral precuneus within the DMN (Fig. 3, Table 2). Within $24 \mathrm{~h}$ of illness onset, the global functional connectivity in the CEN was not statistically different between TGA patients and controls, although a topographical analysis illustrated that higher functional connectivity of the TGA patients was observed on the small areas of the medial prefrontal cortex within the CEN compared to that of the controls.

Sub-analyses for comparison of functional connectivity between hyperacute TGA patients and controls (Fig. 4) showed that hyperacute TGA patients showed increased functional connectivity in the SN, especially on the bilateral insular cortex, which is comparable to that from whole TGA patients. The topographical analysis also demonstrated that hyperacute TGA patients had lower functional connectivity in the bilateral precuneus within the DMN and greater functional connectivity on the right frontal cortex within the CEN, which is consistent with that from whole TGA patients. However, there were no significant differences of functional connectivity in the SN, DMN and the CEN between the post-acute TGA patients and controls.

Longitudinal changes of functional networks in TGA patients. TGA patients did not show greater functional connectivity in the SN $72 \mathrm{~h}$ after TGA onset, while they continued to show decreased functional connectivity in the DMN $72 \mathrm{~h}$ after illness onset (Fig. 5). However, alterations in functional connectivity in the SN and DMN were no longer observed at 3 months after TGA onset. Functional connectivity in the CEN was relatively preserved within $24 \mathrm{~h}$ of illness onset as well as at $72 \mathrm{~h}$ and 3 months after TGA onset. Longitudinal changes of topographical functional connectivity within TGA patients were demonstrated in Figure e-1.

Secondary analyses. Comparison of functional connectivity between TGA patients with hyperacute and those with post-acute phase. When we compared global functional connectivity of the SN, DMN and CEN between TGA patients with hyperacute phase and post-acute phase, there were no significant statistical differences of intrinsic brain network between those two groups (Figure e-2).

Comparison of functional connectivity between TGA patients with hippocampal lesion and those without hippocampal lesion on DWI. There were no significant differences of global functional connectivity in the SN, DMN and CEN between TGA patients with hippocampal lesion $(n=34)$ and those without hippocampal lesion $(\mathrm{n}=16)$ (Figure e-3).

Comparison of functional connectivity between TGA patients with emotional cause and those with other causes. To investigate the effects of precipitating emotional causes on intrinsic brain networks, we compared functional connectivity of the SN, DMN and CEN between TGA patients with emotional causes $(n=26)$ and those with other causes $(n=24)$. However there were no statistical differences of functional connectivity between the two groups (Figure e-4).

\section{Discussion}

Our main finding was that the TGA patients showed greater functional connectivity in the SN compared to controls in the acute phase, which was normalized in $72 \mathrm{~h}$ and 3 months after TGA onset. 


\section{A. Salience Network}

(a)
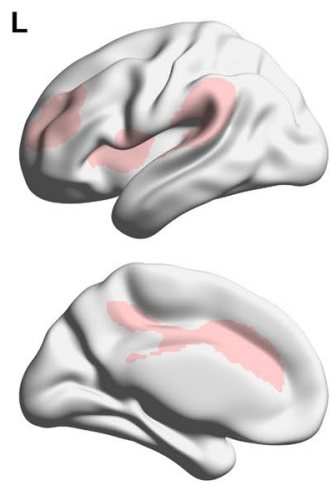
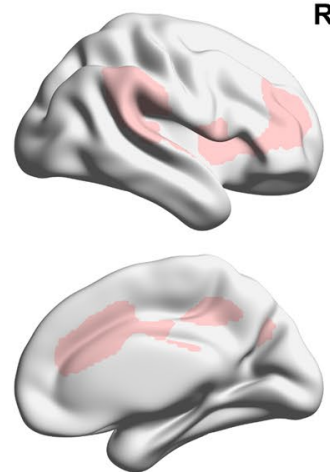

(b)

$\mathbf{R}$

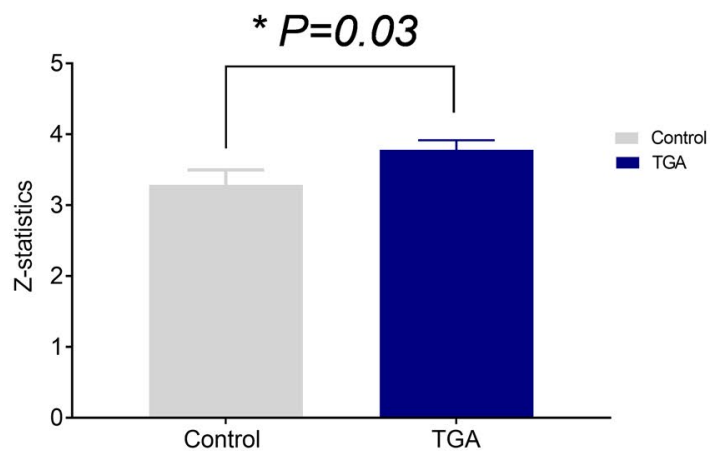

\section{B. Default Mode Network}

(a)
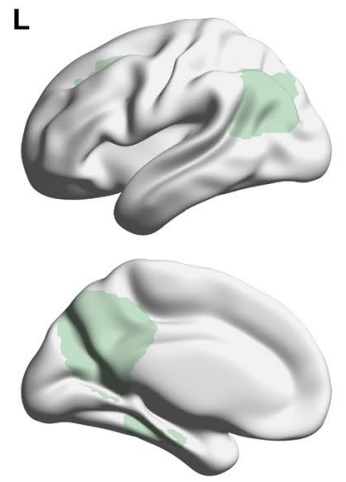
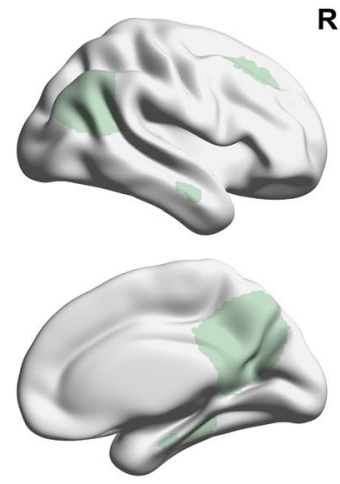

$\mathbf{R}$ (b)

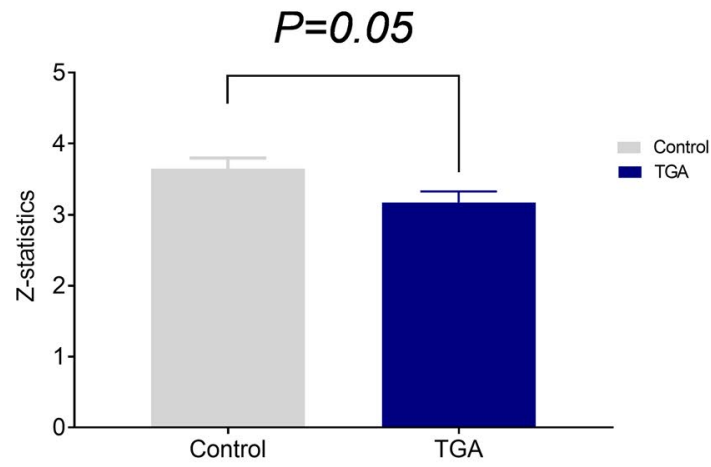

\section{Central Executive Network}

(a)
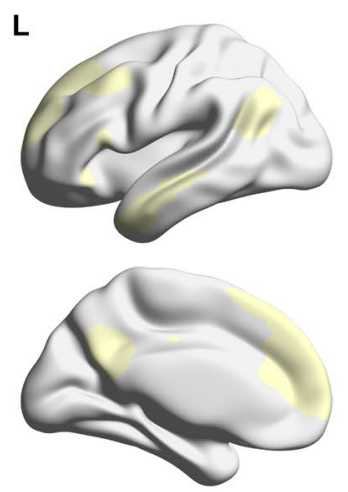

(b)

$\mathbf{R}$

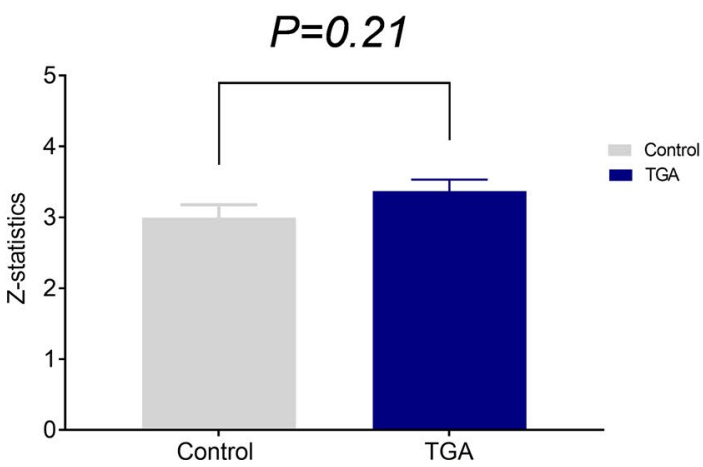

Figure 2. Group differences in the functional intrinsic brain network during the acute phase. A (a) Template for the salience network (SN). (b) Compared to controls, TGA patients during the acute phase of the illness showed higher functional connectivity in the SN. B (a) Template for the default mode network (DMN). (b) Compared to controls, TGA patients demonstrated lower functional connectivity in the DMN during the acute phase, but the statistical significance was equivocal. C (a) Template for the Central Executive Network (CEN). (b) There were no significant group difference of functional connectivity on the CEN between TGA patients and controls. ${ }^{\star} P$ value was adjusted by age and sex. 
In particular, TGA patients with acute phase showed greater functional connectivity in the SN, especially in the bilateral insular cortex. It is well-known that the SN has a role in identifying and integrating interoceptive, autonomic, and emotional information, which leads to detection of the most homeostatically relevant external stimuli to guide behavior ${ }^{9,12,19}$. Therefore, one of the key roles of the SN is switching of the brain networks, leading to the engagement of the CEN and disengagement of the DMN to external stimuli, or the engagement of the $\mathrm{DMN}$ and disengagement of the CEN to internal stimuli ${ }^{9,11}$.

Considering that $96 \%$ of TGA patients reported the precipitating events such as pain, emotional stress or physical distress prior to clinical amnestic symptoms, aberrantly greater functional connectivity of the SN to external salient stimuli might lead to aberrant disengagement of DMN and engagement of the CEN in TGA patients.

In agreement with this hypothesis, patients with TGA had lower functional connectivity in the DMN, especially in the bilateral precuneus. Because the DMN underlies autobiographical and episodic memory ${ }^{20-23}$, our findings are consistent with the previous studies demonstrating lower functional connectivity in the posterior memory network, including the precuneus in TGA patients ${ }^{15,16}$.

In addition, TGA patients continued to show lower functional connectivity in the DMN at $72 \mathrm{~h}$ after illness onset. This finding is consistent with the previous work that showed disruptions in memory networks can be observed between 24 and $48 \mathrm{~h}$ after TGA onset ${ }^{3,6}$. One explanation for delayed abnormalities in the DMN could be that TGA patients might have difficulties with rapid allocation of resources from external salient stimuli in the $\mathrm{SN}$ to internal mental process in the $\mathrm{DMN}^{10}$. Therefore, overactive $\mathrm{SN}$ in TGA patients may cause delayed allocation of resources to internal stimuli for TGA patients, which may cause sustained abnormally lower activities in the DMN for TGA patients. However, further studies are warranted to examine whether alterations in these networks are linked to delayed processing of the brain network.

Unexpectedly, compared to controls, TGA patients did not demonstrate aberrant functional connectivity in the CEN, although the focal areas of increased functional connectivity in the medial prefrontal cortex were noted during the acute phase. Previous studies have shown that TGA patients had preserved working memory and executive functions but prominent impaired episodic impairment during an acute attack of TGA ${ }^{24,25}$, therefore, relatively spared functional connectivity of the CEN during acute phase could explain the preserved cognitive function in working memory and executive function during acute phase of TGA. However, because we did not perform neuropsychological tests during the acute phase of TGA in the current study, further studies involving detailed neuropsychological tests during the acute phase of TGA could help reveal the relationship between preserved CEN and executive function in TGA patients.

It should be noted that previous research with intrinsic brain network of TGA showed lower functional connectivity in the executive network during acute phase ${ }^{17}$, which is contrary to our findings. One of possible explanation for this could be that the current study excluded TGA patients with prior history of any psychiatric illness, whereas $31.3 \%$ of TGA patient in the previous study had a history of psychiatric illness. The lower functional connectivity of the CEN has been reported in patients with psychiatric diseases ${ }^{11,26}$, which might affect the different results.

Interestingly, there were no significant differences of functional connectivity of SN, DMN and CEN between the TGA patients with hyperacute and those with post-acute phase (Figs. 1,2). However, compared to controls, enhanced functional connectivity of the $\mathrm{SN}$ was prominent only in the hyperacute phase, whereas a tendency of lower functional connectivity in the DMN was observed only in the post-acute phase $(P=0.06)$ (Fig. 5). This finding can also support that alterations in functional connectivity of the $\mathrm{SN}$ might precede alterations in the DMN in TGA patients, as mentioned above.

As expected, alterations of global functional connectivity in the $\mathrm{SN}$ and DMN were normalized at the time of the 3-month follow-up examination. This finding is consistent with those of many previous studies that reported that functional changes observed during the acute phase of TGA were normalized at follow-up visits ${ }^{16,27}$. It is also noteworthy that compared to the baseline, TGA patients showed decreased functional connectivity on the right precuneus of the $\mathrm{SN}$ and on the left parahippocampal gyrus of the DMN in $72 \mathrm{~h}$. Considering that such areas of decreased functional connectivity in $72 \mathrm{~h}$ are involved the DMN, it could be also compatible with our findings illustrating that decreased functional connectivity in the DMN was most prominent in $72 \mathrm{~h}$ compared to controls.

There were several limitations to our study. First, we did not include healthy volunteers at the $72 \mathrm{~h}$ and 3-month time point. Instead, we compared patients' data from the $72 \mathrm{~h}$ and 3 -month follow-up visit to the data acquired from healthy controls at the baseline. Second, although there were no significant differences of the baseline clinical characteristics between TGA patients who completed all three follow-up visits compared to those who only participated in two visits, only 22 of 50 patients participated in the 3-month follow-up visit. Third, as mentioned, because we did not perform neuropsychological tests during TGA attacks in the current study, the relationship between alterations in the functional brain network and neuropsychological performance of TGA patients was not explored in this study.

Fourth, it has been known that enhanced SN functional connectivity has been noted in several psychiatric disease such as acute stress, anxiety and post-traumatic stress disorders ${ }^{11,28,29}$. Since $52 \%$ of TGA patients in the current study have reported external salient provoking events such as emotional stress, we cannot totally exclude the possibility that increased functional connectivity in the SN in TGA patients might be related to acute stress reaction. However, when we compared the functional connectivity between TGA patients with emotional stress and those with other causes, there were no significant differences of functional connectivity in the SN between those two groups. Furthermore, the functional connectivity of the DMN, one of well-known vulnerable brain network to acute stress ${ }^{30}$, was not different between the two groups either. It may suggest that such alterations of the functional connectivity in TGA patients might represent characteristic alterations of the intrinsic brain network in TGA patients, not mere reactive alterations to acute stress. However, further studies related to 


\section{A. Salience Network}

(a)
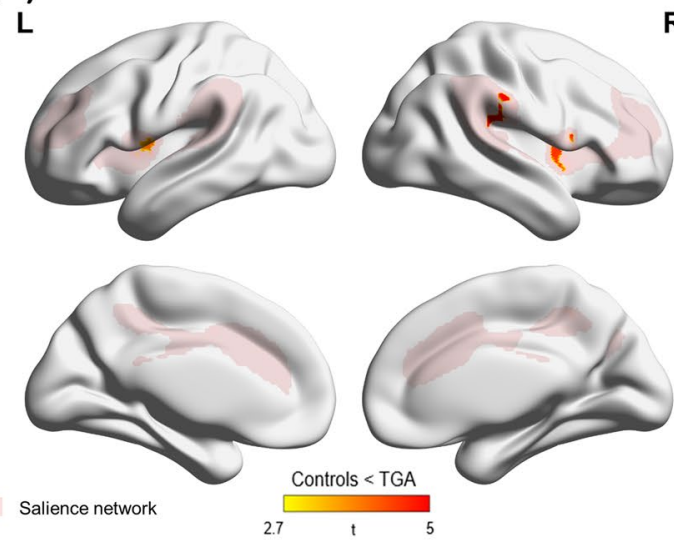

\section{B. Default Mode Network}

(a)
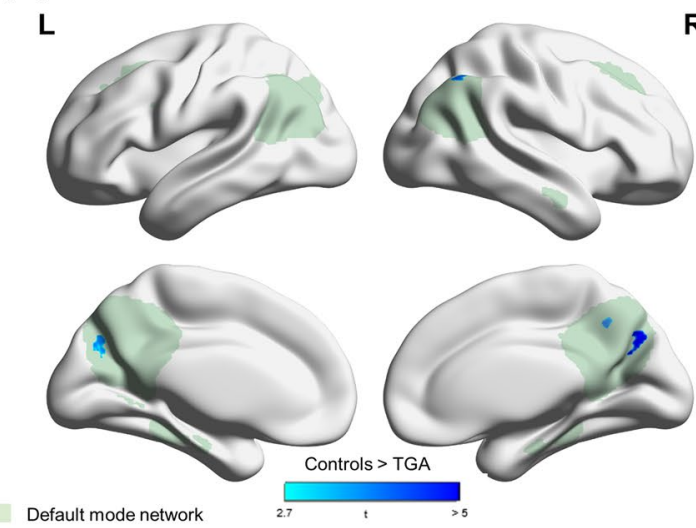

(b)

R L
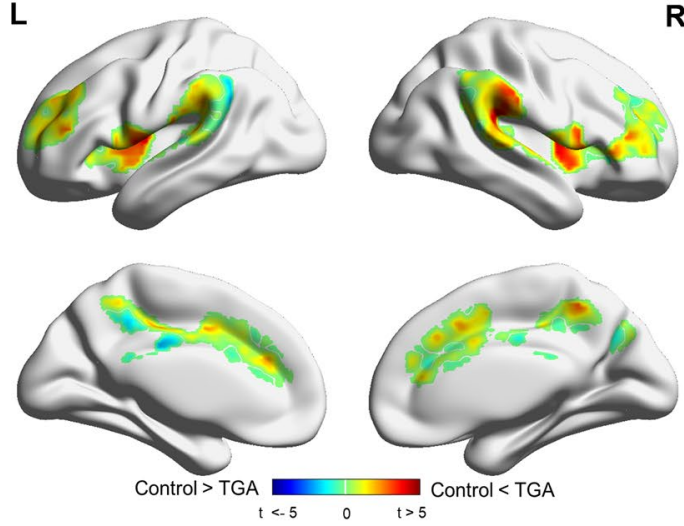

(b)

$\mathbf{R}$
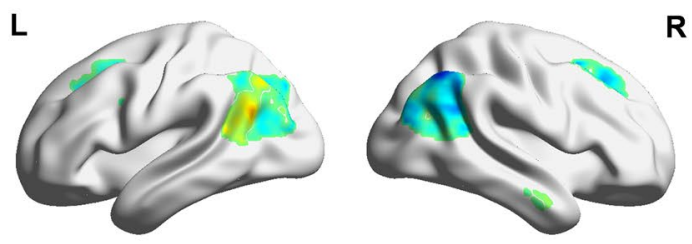

\section{Central Executive Network}

(a)
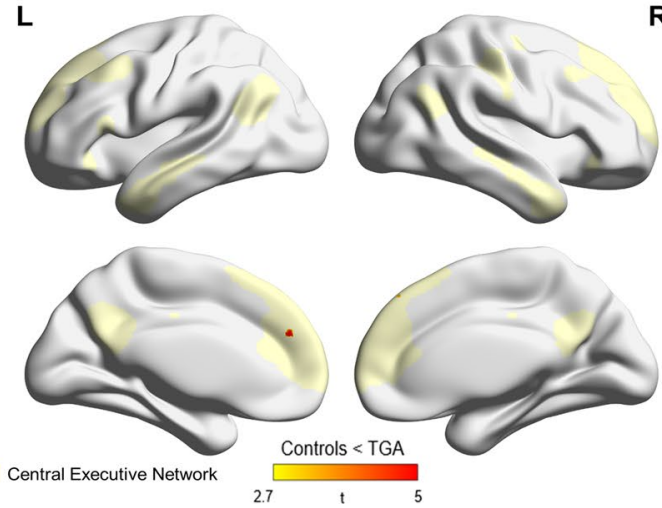

(b)

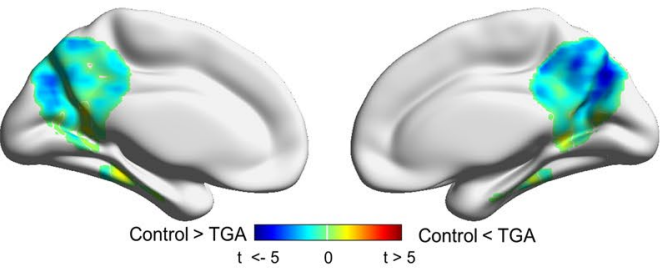

$\mathbf{R}$

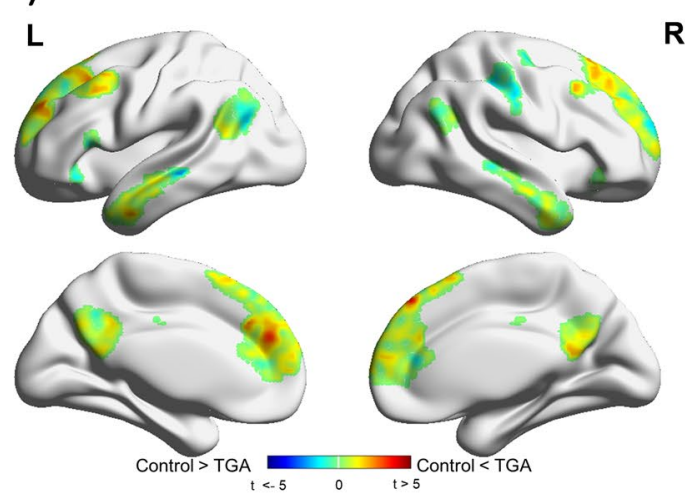


4Figure 3. Topographical differences in the functional intrinsic brain network of controls and TGA patients during the acute phase. A (a) Significant clusters indicating the group differences of functional connectivity in the SN. Compared to controls, TGA patients showed greater functional connectivity in the bilateral insular cortices and operculum within the SN. There were no significant areas that demonstrated greater functional connectivity in the controls within the SN. (b) T-static maps of functional connectivity for the group differences in the SN of TGA patients and controls. B (a) Significant clusters indicating the group differences in functional connectivity in the DMN. TGA patients showed lower functional connectivity in the bilateral precuneus within the DMN compared to controls. However, there were no significant areas that showed lower functional connectivity in the controls within the DMN. (b) T-static maps of functional connectivity for the group differences in the DMN in TGA patients and controls. C (a) Significant clusters indicating the group differences of functional connectivity in the CEN. Topographical analysis illustrated that higher functional connectivity of the TGA patients was observed on the small areas of the medial prefrontal cortex within the CEN compared to that of the controls. (b) T-static maps of functional connectivity for the group differences in the CEN of TGA patients and controls.

functional connectivity according to various precipitating factors could be helpful to elucidate whether a variety of precipitating factors might affect the pathophysiology of TGA differently.

Nonetheless, this is the first study to investigate the intrinsic functional connectivity of TGA patients longitudinally. Our study suggests that greater functional connectivity in the SN and lower functional connectivity in the DMN may be associated with TGA, which are normalized 3 months after illness onset.

\section{Methods}

TGA patients. TGA patients were prospectively recruited from the Ewha Womans University Mokdong Hospital between March 2015 and October 2017. A diagnosis of TGA was based on the following inclusion criteria $^{31,32}$ : the presence of anterograde amnesia, which was witnessed by another individual; no clouding of consciousness or loss of personal identity; the presence of cognitive impairment limited to amnesia; no evidence of neurological abnormalities or epilepsy; no recent history of head trauma or seizures; resolution of amnesia symptoms within $24 \mathrm{~h}$ of onset. Exclusion criteria were as follows: suspected or diagnosed mild cognitive impairment or Alzheimer's disease; severe, major medical, or neurological illnesses; axis I mental illnesses, including current major depressive disorder and lifetime substance use disorders; history of head trauma leading to a loss of consciousness or seizure; any contraindications to MRI; and the use of medications that influence the central nervous system within the past 3 months.

Of 58 TGA patients who visited the emergency department (ER) at the Ewha Womans University Mokdong Hospital, 50 patients agreed to participate in the study. The 6 TGA patients refused to take MRI while 2 patients were excluded due to a history of depression. The 50 TGA patients with acute phase were subdivided into the two groups; hyperacute vs post-acute phase. TGA patients with hyperacute phase referred to those who still had TGA symptoms such as temporal disorientation or repetitive questioning during the baseline MRI scan $(n=26)$, whereas those with post-acute phase refers TGA patients who no longer exhibited repetitive questioning but still displayed memory deficits on MMSE $(n=24)$.

TGA patients underwent MRI and clinical assessment using the Korean version of the Mini Mental Status Examination (K-MMSE) ${ }^{33}$ at ER, within $72 \mathrm{~h}$ and 3 months following TGA onset. All TGA patients were assessed using electrocardiography (ECG), electroencephalography (EEG), and routine laboratory blood tests following admission. All TGA patients underwent a standard cardiovascular examination involving a structured interview to assess vascular and non-vascular risk factors for TGA (hypertension, diabetes, hyperlipidemia, alcohol use, and smoking). A history of cardiovascular, neurological, and psychiatric diseases was recorded (previous cerebrovascular events, TGA, cardiac disease, migraine, epilepsy, psychiatric disease).

Controls. We also included 25 cognitively normal individuals matched according to age and sex without any history of TGA (hereafter referred as "controls"). These controls were spouses of outpatients visiting the Memory Disorder Clinic at Ewha Womans University Mokdong Hospital during the same period and who did not have a history of or current TGA or other neurological or psychiatric illnesses. Controls were selected according to their K-MMSE results ${ }^{33}$ and Seoul Neuropsychological Screening Battery results ${ }^{34}$. The SNSB is an extended neuropsychological battery that includes tests measuring attention, language, calculation, praxis, visuospatial abilities, verbal/visual memory, and executive functioning. Normal cognitive function was defined when individuals scored 1 standard deviation (SD) or more below the population norm on the memory, attention, language, visuospatial, and frontal tests of the SNSB.

Standard protocol approvals, registrations, and patient consents. Written informed consent prior to study participation was obtained from all participants or their legally authorized representatives in accordance with the Declaration of Helsinki., which was repeated in acute TGA patients after symptom resolution. The Institutional Review Board of Ewha Womans University Mokdong Hospital examined and approved the study protocol (EUMC 2015-07-003-016).

Functional magnetic resonance imaging acquisition and analysis. A 3-Tesla Philips Achieva MR scanner (Philips Medical Systems, Noord-Brabant, Netherlands) was used to acquire MR images. The routine MRI protocol for TGA consisted of T1-weighted and T2-weighted imaging, fluid-attenuated inversion recov- 


\begin{tabular}{|c|c|c|c|c|c|}
\hline & \multirow[b]{3}{*}{ Cluster size $\left(\mathrm{mm}^{3}\right)$} & \multirow[b]{3}{*}{ Maximum $t$ value } & \multirow{2}{*}{\multicolumn{3}{|c|}{\begin{tabular}{|l|}
$\begin{array}{l}\text { MNI atlas } \\
\text { coordinates }\end{array}$ \\
$\begin{array}{l}\text { (location of } \\
\text { maximum } t \text {-value) }\end{array}$
\end{tabular}}} \\
\hline & & & & & \\
\hline & & & $x$ & $y$ & $z$ \\
\hline \multicolumn{6}{|l|}{ Salience Network ( $\uparrow$ in TGA) } \\
\hline Fronto-parietal operculum (L) & 2424 & 4.86 & 48 & -28 & 20 \\
\hline Anterior insular (R) & 2032 & 4.55 & 38 & 0 & 10 \\
\hline Anterior insular (L) & 896 & 4.5 & -36 & -4 & 8 \\
\hline Frontal operculum (L) & 744 & 4.11 & -52 & -4 & 12 \\
\hline Superior frontal (L) & 360 & 3.86 & -62 & -26 & 20 \\
\hline \multicolumn{6}{|l|}{ Default Mode Network ( $\downarrow$ in TGA) } \\
\hline Precunues(R) & 1320 & 4.87 & 16 & -70 & 38 \\
\hline Angular gyrus(R) & 728 & 3.71 & 44 & -60 & 44 \\
\hline Precuneus(L) & 496 & 3.61 & -14 & -72 & -28 \\
\hline Precunues(R) & 440 & 3.82 & 8 & -54 & 42 \\
\hline \multicolumn{6}{|c|}{ Central Executive Network ( $\uparrow$ in TGA) } \\
\hline Medial Prefrontal (L) & 336 & 3.63 & -4 & 44 & 26 \\
\hline
\end{tabular}

Table 2. Cluster information of different functional connectivity between the controls and the TGA patients. Significant difference of clusters in the default mode, salience and central executive network between the controls and TGA patients. Regions of decreased or increased connectivity in the TGA group were shown at the cluster-corrected $P<0.05$.

ery, and conventional gradient-echo images in the transverse plane and three-dimensional (3D) time-of-flight angiography of the intracranial region. Diffusion-weighted imaging (DWI) was performed at $72 \mathrm{~h}$ after onset to detect high signal intensities on the hippocampus of TGA patients. DWI was performed in the transverse and coronal planes covering the entire brain with $\mathrm{b}=1000\left(\mathrm{~s} / \mathrm{mm}^{2}\right)$ and $3-\mathrm{mm}$ slice thickness.

For functional network analysis, structural images were acquired using a 3D T1-weighted magnetizationprepared rapid gradient-echo imaging sequence with the following acquisition parameters: repetition time (TR), $9.9 \mathrm{~ms}$; echo time (TE), $4.6 \mathrm{~ms}$; flip angle (FA), $8^{\circ}$; field of view (FOV), $240 \times 240 \mathrm{~mm}^{2}$; slice thickness, $1 \mathrm{~mm}$; number of excitations (NEX), 1; number of slices, 160; and orientation, sagittal. Resting state functional images were acquired using an echo planar imaging sequence with the following parameters: TR, 3,000 ms; TE, $35 \mathrm{~ms}$; FA, $90^{\circ}$; FOV, $220 \times 220 \mathrm{~mm}^{2}$; slice thickness, $4.0 \mathrm{~mm}$; number of slices, 35; and the duration of scan time, 5:09. Participants were instructed to think about nothing in particular, to keep their eyes closed, and to stay awake. An automated shimming procedure was used to reduce the influence of field inhomogeneities before performing functional MRI scans.

Functional imaging data were pre-processed using FMRIB Software Library tools (FSL, http://www.fmrib.ox. ac.uk/fsl). Standard pre-processing steps were conducted, including motion correction using multi-resolution rigid body co-registration, brain extraction using the FSL Brain Extraction Tool (BET $)^{35}$, spatial smoothing using a Gaussian kernel $(5 \mathrm{~mm})$, and high-pass filtering $(0.01 \mathrm{~Hz})$. Functional imaging data for each individual were co-registered with the corresponding T1-weighted structural image. These co-registered images were then normalized to the Montreal Neurological Institute (MNI) atlas using affine registration (12 degrees of freedom). The pre-processed images were then concatenated in the temporal dimension to create a single four-dimensional (4D) dataset. There were no significant differences in head motion parameters of TGA patients and healthy volunteers for absolute head motion (TGA group, $0.224 \pm 0.233 \mathrm{~mm}$; controls, $0.161 \pm 0.020 ; t=-1.28 ; P=0.203$ ) or relative head motion (TGA group, $0.086 \pm 0.040 \mathrm{~mm}$; Controls, $0.080 \pm 0.033 \mathrm{~mm} ; t=-0.61 ; P=0.55$ ).

Single-subject independent component analyses (ICA) were performed using multivariate exploratory linear optimized decomposition into independent components (MELODIC) ${ }^{36}$. Then, the ICA-based Xnoiseifier (FIX) of the FMRIB was used to remove components corresponding to structural artifacts from each functional image data set ${ }^{37}$. A group ICA, which is a model-free and data-driven approach, was performed to separate 4D functional MRI data into a set of independent one-dimensional (1D) time-series and related 3D spatial maps ${ }^{36,38}$ based on the unified group from the whole participants (50 TGA patients and 25 controls). In the current study, functional imaging data were decomposed into 20 independent components (dimensionality $=20$ ) using a temporal concatenation approach. Consequently, 10 components were classified as anatomically and functionally meaningful resting state networks (RSNs) corresponding to functional networks previously described ${ }^{38}$, while 10 components were classified as artifacts following the visual inspection of an experienced researcher (G.H.). Of the 10 RSNs that were identified, we selected three RSNs of interest, including the SN, DMN, and CEN. The three aforementioned RSNs of interest were used in all subsequent analyses. Component information and spatial maps of the available components with a threshold of $\mathrm{z}=3.1(P=0.001)$ are presented in Figure e-5. We also obtained a group ICA map with 26 hyperacute TGA patients and 25 controls, which was similar to that of whole participants (Figure e-6).

A dual linear regression approach was applied to identify subject-specific time courses and spatial maps ${ }^{36}$. The group ICA map for the SN, DMN and CEN based on the whole participants was used to perform linear 


\section{A. Salience Network}

(a)

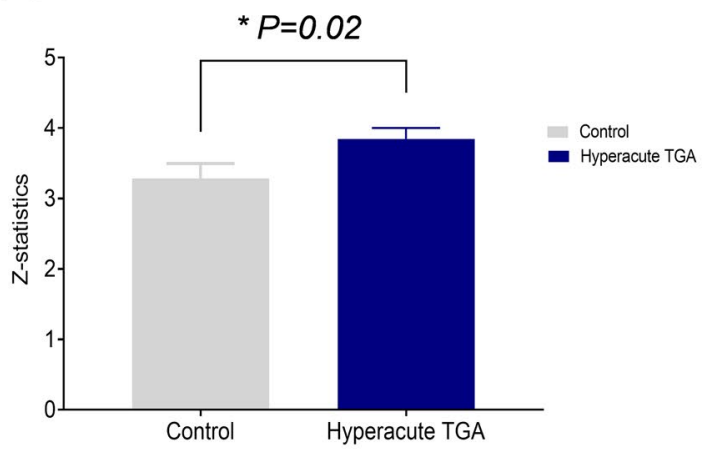

(b)

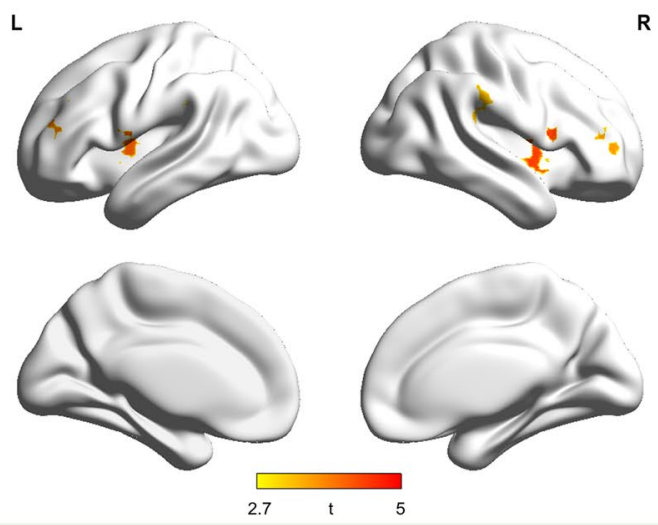

\section{B. Default mode network}

(a)

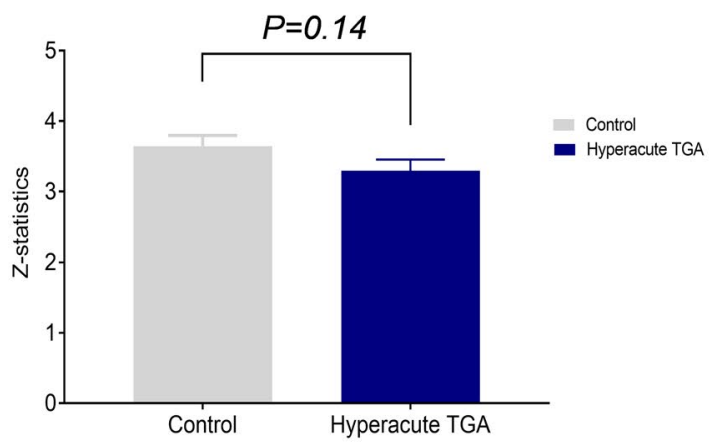

(b)

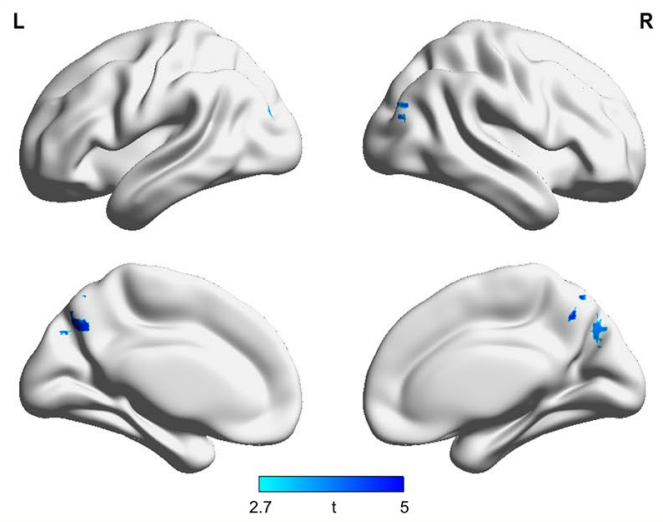

\section{Central Executive Network}

(a)

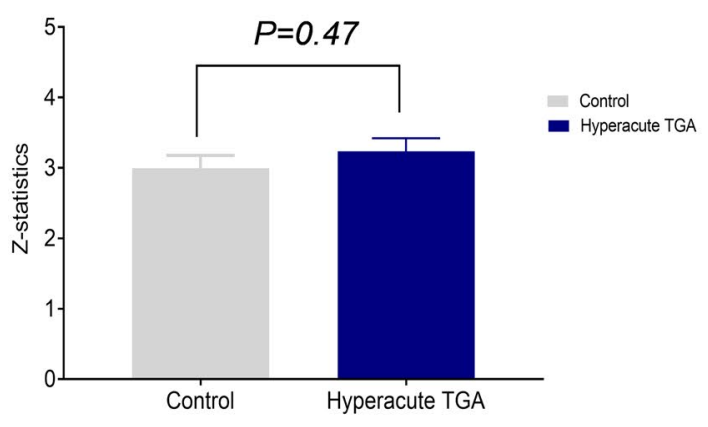

(b)

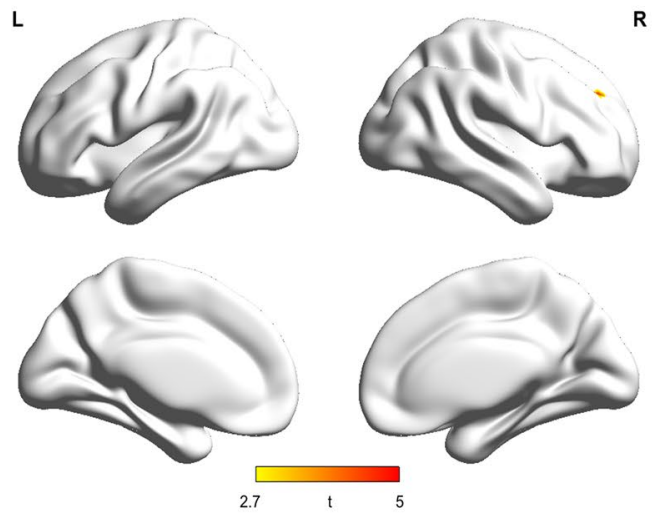

Figure 4. Global and topographical differences in the functional intrinsic brain network between the controls and hyperacute TGA patients. A (a) Compared to controls, TGA patients during the hyperacute phase showed higher functional connectivity in the SN. (b) Compared to controls, TGA patients showed greater functional connectivity in the bilateral insular and frontal cortices within the SN. B (a) There were no significant group difference of functional connectivity on the DMN between hyperacute TGA patients and controls. (b) TGA patients with hyperacute phase showed lower functional connectivity in the bilateral precuneus and right inferior parietal cortex within the DMN compared to controls. C (a)There were no significant group difference of functional connectivity on the CEN between hyperacute TGA patients and controls. (b) Topographical analysis illustrated that higher functional connectivity of the TGA patients with hyperacute phase was observed on the small areas of the right dorsolateral prefrontal cortex within the CEN compared to that of the controls. 


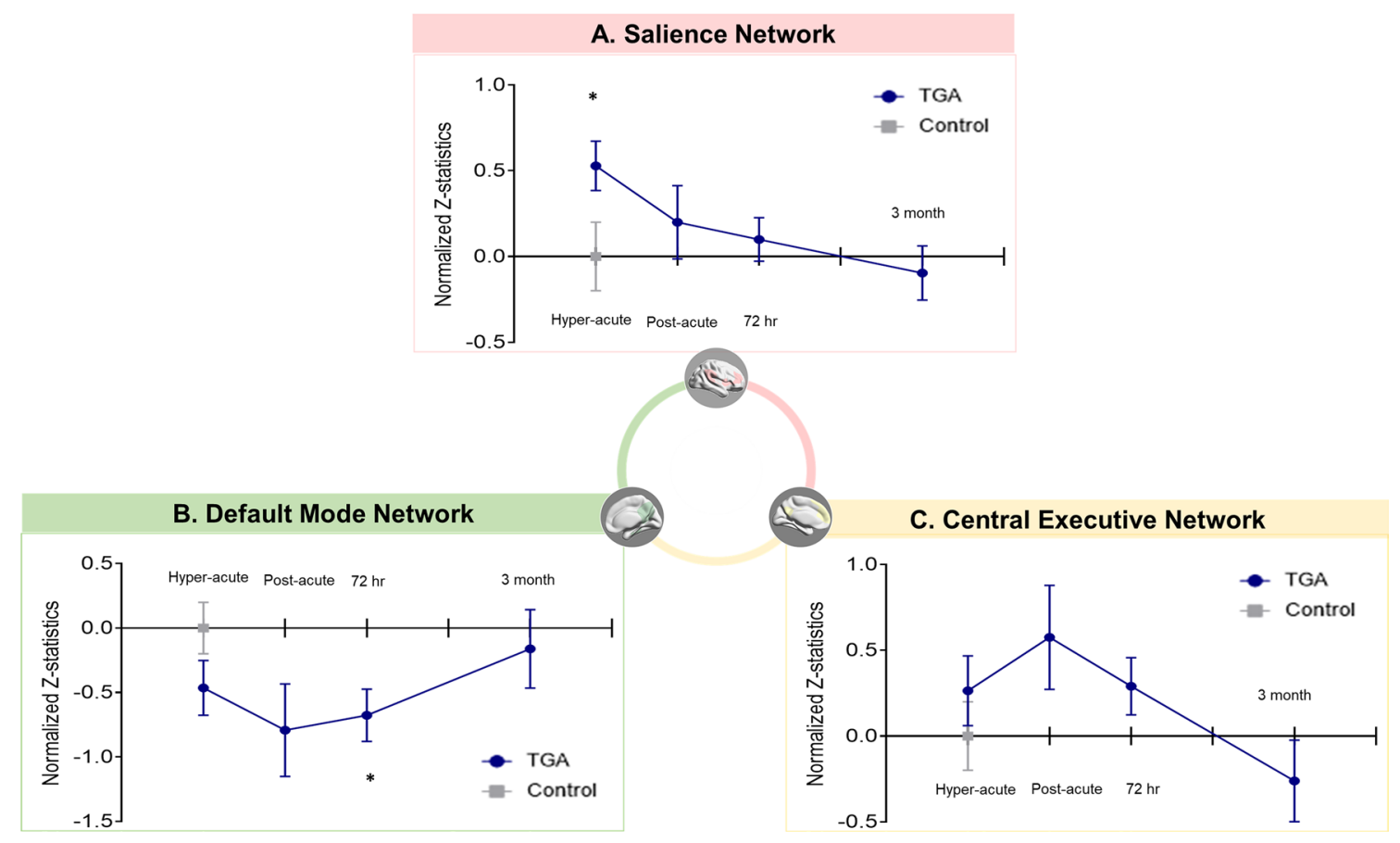

Figure 5. Longitudinal changes of functional connectivity in the intrinsic brain network among TGA patients. (A) TGA patients with hyperacute phase showed greater functional connectivity in the SN whereas, those with post-acute phase showed no statistical different functional connectivity in the SN compared to controls. TGA patients did not show greater functional connectivity in the $\mathrm{SN}$ at $72 \mathrm{~h}$ after TGA onset. Additionally, alterations in functional connectivity in the SN were no longer observed at 3 months after TGA onset. (B) Compared to controls, there were no significant differences of DMN in TGA patients with hyperacute phase or in those with post-acute phase. TGA patients continue to show decreased functional connectivity in the DMN $72 \mathrm{~h}$ following TGA onset, whereas alterations in functional connectivity in the DMN were no longer shown 3 months following TGA onset. (C) Functional connectivity in the CEN was relatively preserved within $24 \mathrm{~h}$ of illness onset as well as at $72 \mathrm{~h}$ and 3 months after TGA onset.

model fit against each subject's functional MRI and to create the average time course within the SN, DMN and CEN for each subject (spatial regression). The time courses of the subjects were variance-normalized and then regressed against the subject's functional MRI data to create subject-specific spatial maps for the SN, DMN, and CEN (temporal regression).

Statistical analysis. Demographic and clinical characteristics were compared between patients with TGA and controls using independent t tests for continuous variables and chi-square tests or Fisher's exact tests for categorical variables, respectively.

Group differences in functional connectivity were analyzed using a voxel-wise $t$ test. Voxel-wise analyses were performed using an ROI mask of the SN, DMN and CEN. The results were corrected for multiple comparisons using the Monte Carlo simulation adjusting for 10,000 iterations implemented in the AlphaSim utility (http:// afni.nimh.nih.gov/pub/dist/doc/program_help/AlphaSim.html). A threshold derived from a combination of $P<0.01$ and a cluster size of a minimum of $336 \mathrm{~mm}^{3}$ was used to correct for multiple comparisons at $P<0.05$. An alpha-level of $P<0.05$ (two-tailed) was considered statistically significant for all analyses. The $Z$ values that represent the functional connectivity of the corresponding RSNs, were extracted from the ROI masks of the SN, $\mathrm{DMN}$ and CEN, which was then used for further analyses. The $\mathrm{Z}$ values of each three functional connectivity in TGA patients were converted to normalized $z$-values using the baseline mean scores and standard deviation of the controls. The normalized z-values were used to show the longitudinal changes of RSNs in TGA patients.

General linear model was performed to examine the group differences in functional connectivity of the corresponding RSNs after adjusting age and sex, while a linear mixed- effect model was employed to estimate the time effects on the RSNs in TGA patients. This model included functional connectivity of the RSNs as linear terms and within-individual variations as random effects. Age and gender were included as covariates.

Data were analyzed using Stata v13.1 (StataCorp., College Station, TX).

\section{Data availability}

Data and statistical algorithms used for analyses are available from the corresponding author on reasonable request. 
Received: 31 July 2020; Accepted: 30 August 2021

Published online: 18 October 2021

\section{References}

1. Quinette, P. et al. What does transient global amnesia really mean?-review of the literature and thorough study of 142 cases. Brain J. Neurol. 129, 1640-1658. https://doi.org/10.1093/brain/awl105 (2006).

2. Fisher, C. M. \& Adams, R. D. Transient global amnesia. Acta Neurologica Scandinavica. Supplementum 40, 1 (1964).

3. Bartsch, T. \& Deuschl, G. Transient global amnesia: functional anatomy and clinical implications. The Lancet. Neurol. 9, $205-214$. https://doi.org/10.1016/s1474-4422(09)70344-8 (2010).

4. Bartsch, T. et al. Selective affection of hippocampal CA-1 neurons in patients with transient global amnesia without long-term sequelae. Brain J. Neurol. 129, 2874-2884. https://doi.org/10.1093/brain/awl248 (2006).

5. Bartsch, T., Alfke, K., Deuschl, G. \& Jansen, O. Evolution of hippocampal CA-1 diffusion lesions in transient global amnesia. Ann. Neurol. 62, 475-480. https://doi.org/10.1002/ana.21189 (2007).

6. Sedlaczek, O. et al. Detection of delayed focal MR changes in the lateral hippocampus in transient global amnesia. Neurology $\mathbf{6 2}$, 2165-2170. https://doi.org/10.1212/01.wnl.0000130504.88404.c9 (2004).

7. Fransson, P. Spontaneous low-frequency BOLD signal fluctuations: an fMRI investigation of the resting-state default mode of brain function hypothesis. Hum. Brain Mapp 26, 15-29. https://doi.org/10.1002/hbm.20113 (2005).

8. Bonnelle, V. et al. Salience network integrity predicts default mode network function after traumatic brain injury. Proc. Natl. Acad. Sci. U S A 109, 4690-4695. https://doi.org/10.1073/pnas.1113455109 (2012).

9. Menon, V. \& Uddin, L. Q. Saliency, switching, attention and control: a network model of insula function. Brain Struct. Funct. 214, 655-667 (2010).

10. Jilka, S. R. et al. Damage to the salience network and interactions with the default mode network. J. Neurosci. 34, 10798-10807 (2014).

11. Menon, V. Large-scale brain networks and psychopathology: a unifying triple network model. Trends Cogn. Sci. 15, 483-506. https://doi.org/10.1016/j.tics.2011.08.003 (2011).

12. Seeley, W. W. et al. Dissociable intrinsic connectivity networks for salience processing and executive control. J. Neurosci. 27, 2349-2356. https://doi.org/10.1523/jneurosci.5587-06.2007 (2007).

13. Gusnard, D. A., Akbudak, E., Shulman, G. L. \& Raichle, M. E. Medial prefrontal cortex and self-referential mental activity: relation to a default mode of brain function. Proc. Natl. Acad. Sci. U S A 98, 4259-4264. https://doi.org/10.1073/pnas.071043098 (2001).

14. Buckner, R. L., Andrews-Hanna, J. R. \& Schacter, D. L. The brain's default network: anatomy, function, and relevance to disease. Ann. N. Y. Acad. Sci. 1124, 1-38. https://doi.org/10.1196/annals.1440.011 (2008).

15. Park, Y. H. et al. Disruption of the posterior medial network during the acute stage of transient global amnesia: a preliminary study. Clin. EEG Neurosci. https://doi.org/10.1177/1550059414543684 (2014).

16. Peer, M. et al. Reversible functional connectivity disturbances during transient global amnesia. Ann. Neurol. 75, 634-643. https:// doi.org/10.1002/ana.24137 (2014).

17. Zidda, F. et al. Resting-state connectivity alterations during transient global amnesia. NeuroImage. Clin. 23, 101869. https://doi. org/10.1016/j.nicl.2019.101869 (2019).

18. Quinette, P. et al. What does transient global amnesia really mean? Review of the literature and thorough study of 142 cases. Brain J. Neurol. 129, 1640-1658 (2006).

19. Sridharan, D., Levitin, D. J. \& Menon, V. A critical role for the right fronto-insular cortex in switching between central-executive and default-mode networks. Proc. Natl. Acad. Sci. 105, 12569-12574 (2008).

20. Qin, P. \& Northoff, G. How is our self related to midline regions and the default-mode network?. Neuroimage 57, 1221-1233 (2011).

21. Ranganath, C. \& Ritchey, M. Two cortical systems for memory-guided behaviour. Nat. Rev. Neurosci. 13, 713-726. https://doi.org/ $10.1038 / \mathrm{nrn} 3338(2012)$.

22. Bartsch, T. \& Butler, C. Transient amnesic syndromes. Nat. Rev. Neurol. 9, 86-97. https://doi.org/10.1038/nrneurol.2012.264 (2013).

23. Philippi, C. L., Tranel, D., Duff, M. \& Rudrauf, D. Damage to the default mode network disrupts autobiographical memory retrieval. Soc. Cogn. Affect. Neurosci. 10, 318-326. https://doi.org/10.1093/scan/nsu070 (2015).

24. Hodges, J. R. Semantic memory and frontal executive function during transient global amnesia. J. Neurol Neurosurg. Psychiatry 57, 605-608. https://doi.org/10.1136/jnnp.57.5.605 (1994).

25. Quinette, P. et al. Working memory and executive functions in transient global amnesia. Brain J. Neurol. 126, 1917-1934. https:// doi.org/10.1093/brain/awg201 (2003).

26. Banich, M. T. et al. Cognitive control mechanisms, emotion and memory: a neural perspective with implications for psychopathology. Neurosci. Biobehav. Rev. 33, 613-630. https://doi.org/10.1016/j.neubiorev.2008.09.010 (2009).

27. Jang, J. W. et al. Longitudinal cerebral perfusion change in transient global amnesia related to left posterior medial network disruption. PLoS ONE 10, e0145658. https://doi.org/10.1371/journal.pone.0145658 (2015).

28. Wang, X., Zhang, W., Sun, Y., Hu, M. \& Chen, A. Aberrant intra-salience network dynamic functional connectivity impairs largescale network interactions in Schizophrenia. Neuropsychologia https://doi.org/10.1016/j.neuropsychologia.2016.11.003 (2016).

29. Pannekoek, J. N. et al. Aberrant limbic and salience network resting-state functional connectivity in panic disorder without comorbidity. J. Affect. Disord. 145, 29-35 (2013).

30. Zhang, W. et al. Acute stress alters the 'default' brain processing. Neuroimage 189, 870-877. https://doi.org/10.1016/j.neuroimage. 2019.01.063 (2019).

31. Hodges, J. \& Warlow, C. Syndromes of transient amnesia: towards a classification. A study of 153 cases. J. Neurol. Neurosurg. Psychiatry 53, 834-843 (1990).

32. Caplan, L. Transient Global Amnesia Handbook of Clinical Neurology. Vol. 45 (1998).

33. Han, C. et al. An adaptation of the Korean mini-mental state examination (K-MMSE) in elderly Koreans: demographic influence and population-based norms (the AGE study). Arch. Gerontol. Geriatr. 47, 302-310 (2008)

34. Ahn, H. J. et al. Seoul Neuropsychological Screening Battery-dementia version (SNSB-D): a useful tool for assessing and monitoring cognitive impairments in dementia patients. J. Korean Med. Sci. 25, 1071-1076. https://doi.org/10.3346/jkms.2010.25.7.1071 (2010).

35. Jenkinson, M., Bannister, P., Brady, M. \& Smith, S. Improved optimization for the robust and accurate linear registration and motion correction of brain images. Neuroimage 17, 825-841. https://doi.org/10.1016/s1053-8119(02)91132-8 (2002).

36. Beckmann, C. F., DeLuca, M., Devlin, J. T. \& Smith, S. M. Investigations into resting-state connectivity using independent component analysis. Philos. Trans. R. Soc. London. Ser. B Biol. Sci. 360, 1001-1013. https://doi.org/10.1098/rstb.2005.1634 (2005).

37. Griffanti, L. et al. ICA-based artefact removal and accelerated fMRI acquisition for improved resting state network imaging. Neuroimage 95, 232-247. https://doi.org/10.1016/j.neuroimage.2014.03.034 (2014).

38. Smith, S. M. et al. Correspondence of the brain's functional architecture during activation and rest. Proc. Natl. Acad. Sci. U S A 106, 13040-13045. https://doi.org/10.1073/pnas.0905267106 (2009). 


\title{
Author contributions
}

G.H.K., MD, PhD : Conception and design, Data analysis and interpretation and Manuscript writing. B.R.K., PhD, M.Y.C., MD and K.D.P., MD, PhD: Collection and assembly of data. S.M.L., MD, PhD: Data analysis and interpretation. J.H.J., MD, PhD: Conception and design, Administrative support, Data analysis and interpretation, Manuscript writing and Final approval of manuscript.

\section{Funding}

This study was supported by a National Research Foundation of Korea (NRF) grant funded by the Ministry of Science and ICT for convergent research in a development program for convergence R\&D of science and technology and liberal arts (NRF- 2020M3C1B6112160), an Original Technology Research Program for Brain Science through the NRF funded by the Ministry of Science and ICT (NRF-2018M3C7A1057137), Basic Science Research Program through the National Research Foundation of Korea(NRF) funded by the Ministry of Education(NRF-2020R1I1A1A01072283), the Korea Health Technology R\&D Project through the Korea Health Industry Development Institute (KHIDI) funded by the Ministry of Health \& Welfare of Korea (HI18C0460, HU20C0271 and HU21C0016), and an Ewha alumni medical research grant.

\section{Competing interests}

All authors declare that this research was conducted in the absence of any commercial or financial relationships that could be construed as a potential conflict of interest.

\section{Additional information}

Supplementary Information The online version contains supplementary material available at https://doi.org/ 10.1038/s41598-021-97842-y.

Correspondence and requests for materials should be addressed to J.H.J.

Reprints and permissions information is available at www.nature.com/reprints.

Publisher's note Springer Nature remains neutral with regard to jurisdictional claims in published maps and institutional affiliations.

\begin{abstract}
(c) (i) Open Access This article is licensed under a Creative Commons Attribution 4.0 International License, which permits use, sharing, adaptation, distribution and reproduction in any medium or format, as long as you give appropriate credit to the original author(s) and the source, provide a link to the Creative Commons licence, and indicate if changes were made. The images or other third party material in this article are included in the article's Creative Commons licence, unless indicated otherwise in a credit line to the material. If material is not included in the article's Creative Commons licence and your intended use is not permitted by statutory regulation or exceeds the permitted use, you will need to obtain permission directly from the copyright holder. To view a copy of this licence, visit http://creativecommons.org/licenses/by/4.0/.
\end{abstract}

(c) The Author(s) 2021 